\title{
Long-Range Order and Segregation in Semiconductor Superlattices
}

\author{
S. Ciraci ${ }^{(a)}$ and Inder P. Batra ${ }^{(b)}$ \\ IBM Zurich Research Laboratory, CH-8803 Rüschlikon, Switzerland \\ (Received 8 August 1986)
}

\begin{abstract}
Results of self-consistent energy-minimization calculations provide strong evidence that the ordered phases in epitaxially grown $\mathrm{Ga}_{1-x} \mathrm{Al}_{x}$ As and strained $\mathrm{Si}_{1-x} \mathrm{Ge}_{x}$ alloys are metastable, in the sense that segregation into constituents is favored. We show that the long-range order in intermediate metastable structures leads to significant changes in the electronic properties of semiconductor superlattices. Segregation gives rise to micro-quantum-wells with staggered band lineup and multiple confined states in the potential barrier.
\end{abstract}

PACS numbers: $73.30 .+\mathrm{y}, 73.40 . \mathrm{Lq}$

Recently a novel aspect of the semiconductor superlattices has been pointed out by Kuan et al. ${ }^{1}$ They reported the observation of long-range order in $\mathrm{Ga}_{1-x} \mathrm{Al}_{x} \mathrm{As}$ alloys grown on $\mathrm{GaAs}(110)$ and (100) substrates. Subsequently, Ourmazd and Bean ${ }^{2}$ presented evidence for a neostructural order-disorder transition in the $\mathrm{Si}_{1-x} \mathrm{Ge}_{x} /$ $\mathrm{Si}(001)$ strained superlattice system. In this Letter, we present important stability considerations for ordering, and novel effects on the electronic structure. We find the neostructural phase transition proposed $^{2}$ in the $\mathrm{Si}-\mathrm{Ge}$ system lowers the total energy. However, this structure is only a local minimum on the Born-Oppenheimer surface, and a deeper minimum corresponds to the segregation of $\mathrm{Si}$ and strained $\mathrm{Ge}(001)$ layers. The ordered $\mathrm{GaAlAs}_{2}$ ternary phase is less stable relative to the disproportion into constituent compounds, indicating that during epitaxial growth, the domains with GaAs and AlAs compounds are segregated from the $\mathrm{Ga}_{1-x} \mathrm{Al}_{x} \mathrm{As}$ alloy. The segregation into constituents produces micro-quantum-wells and confined states in the alloy. The band lineup in small period superlattices is found to be different from large superlattices.

We arrived at these conclusions by extensive totalenergy minimization and electronic-structure calculations using the self-consistent-field (SCF) pseudopotential method. ${ }^{3}$ The use of local-density-functional theory in successfully obtaining conduction-band-related properties has been demonstrated ${ }^{4}$ (despite the low estimate for the band gap). Energetics are compared in an internally consistent manner by the use of calculated equilibrium lattice constants of bulk $\mathrm{Si}, \mathrm{Ge}, \mathrm{GaAs}$, and AlAs with the same energy cutoffs.

In the stability analysis of the $\mathrm{Si}-\mathrm{Ge}$ system, one must consider the strain ${ }^{5}$ imposed by the $\mathrm{Si}(001)$ substrate, which yields a tetragonal distortion in the pseudomorphically grown overlayer. Accordingly, a neostructural change must preserve the registry of the substrate. Another important aspect is that a meaningful comparison of the total energy can be made only among similar supercells. The supercell shown in Fig. 1 satisfies these
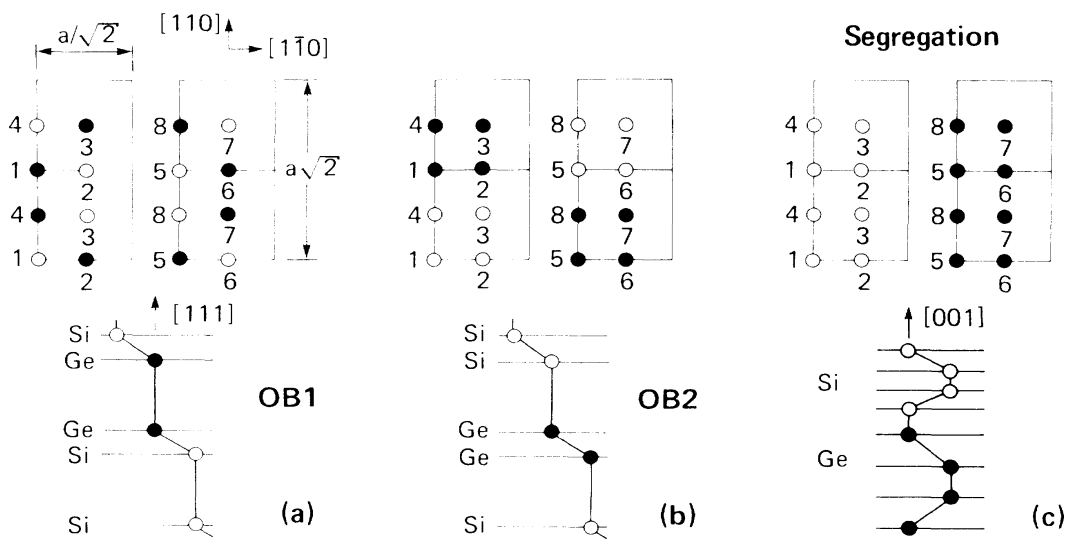

FIG. 1. (a) RH SiGe OB1, (b) RH SiGe OB2, (c) segregated SiGe(001) ordered structures consisting of eight (001) - (2×1) layers with eight $\mathrm{Si}$ and eight $\mathrm{Ge}$ atoms. Numerals identify the layers. For clarity, the first and second sets of four $(001)-(2 \times 1)$ layers are shown separately. The ordering of $\mathrm{OB} 1$ and $\mathrm{OB} 2$ structures along [111] are shown at the bottom of panels (a) and (b), respectively. The bottom panel in (c) of the lowest-energy segregated structure depicts three interplanar distances along [001]; $d(\mathrm{Ge}-$ $\mathrm{Ge})>d(\mathrm{Si}-\mathrm{Ge})>d(\mathrm{Si}-\mathrm{Si})$. 
constraints. In addition, it is also appropriate for the study of totally segregated $\mathrm{Si}_{4} \mathrm{Ge}_{4}(001)$, the epitaxial zinc-blende (ZB) structure, and to some extent the "quasi" disordered $\mathrm{Si}_{0.5} \mathrm{Ge}_{0.5}$ alloy.

The ordered phase proposed by Ourmazd and Bean ${ }^{2}$ has two equipolar rhombohedral ( $\mathrm{RH}$ ) variants, both allowing bilayer segregation normal to the [111] direction. In the first one, denoted by $\mathrm{OB} 1, \mathrm{Si}(\mathrm{Ge})$ has three heteropolar bonds, and pairs of widely spaced planes are occupied by atoms of the same kind [Fig. 1(a)] leading to $\mathrm{Si}-\mathrm{Ge}-\mathrm{Ge}-\mathrm{Si}-\mathrm{Si}$. In $\mathrm{OB} 2, \mathrm{Si}(\mathrm{Ge})$ has three homopolar bonds, and pairs of widely spaced planes are occupied by atoms of the opposite kind [Fig. 1(b)] leading to $\mathrm{Si}-\mathrm{Si}-\mathrm{Ge}-\mathrm{Ge}-\mathrm{Si}$. Recall that in the ZB structure (each atom has four heteropolar bonds) no bilayer segregation normal to the [111] is possible because of the arrangement $\mathrm{Si}-\mathrm{Ge}-\mathrm{Si}-\mathrm{Ge}-\mathrm{Si}$. The disorder of the $\mathrm{Si}_{0.5} \mathrm{Ge}_{0.5}$ alloy is simulated by our creating supercells with altered coordination sequence of atoms and averaging the total energies. The strain is introduced by the use of the equilibrium lattice constant of $\mathrm{Si}$ for the lateral lattice parameters in all supercells. The preferential accommodation of strain by Ge layers is consistent with experimental observations ${ }^{2}$ and also with higher values (see, for example, Keating ${ }^{5}$ ) the force constants for $\mathrm{Si}$ relative to $\mathrm{Ge}$. The interlayer spacings between $\mathrm{Si}$ and strained $\mathrm{Ge}(001)$ layers, and strained $\mathrm{Ge}-\mathrm{Ge}(001)$ layers are determined by total-energy minimization. The interlayer spacing between $\mathrm{Si}_{0.5} \mathrm{Ge}_{0.5}$ alloy layers are obtained by scaling the $x$-ray-measured vertical strain ${ }^{6}$ (perpendicular to substrate) with respect to the calculated equilibrium lattice constants. That such an approach is accurate has been justified by the work of Van de Walle and Martin. ${ }^{4}$

SCF total-energy calculations ${ }^{3}$ have been carried out for these supercells with a 1200-plane-wave basis set - 900 of which were treated exactly; the remaining 300 were included by use of Löwdin's partition scheme. Results yield that the RH-ordered OB1 structure has slightly lower energy ( $1 \mathrm{mRy} / \mathrm{cell})$ relative to the "averaged" disordered structure, and OB2 has higher energy relative to $\mathrm{OB} 1$. The energy of the optimized $\mathrm{OB} 1$ (without trigonal distortion along the [111] direction) structure calculated in the primitive (four atoms) unit cell is $0.75 \mathrm{mRy} /$ atom higher than the average equilibrium energy of bulk $\mathrm{Si}$ and Ge. This result is in fair agreement with the value $(\sim 0.5 \mathrm{mRy} / \mathrm{atom})$ obtained by Martins and Zunger. ${ }^{7}$ The OB1 structure is definitely more stable relative to the alloy (when both OBI and alloy are pseudomorphic with the Si substrate). However, the OB1 structure is unstable (even in the absence of any strain; the introduction of strain would destabilize it even further) relative to bulk $\mathrm{Si}$ and Ge. The $\mathrm{ZB}-\mathrm{SiGe}$ structure has higher energy ${ }^{7}$ still. The relative stability of the RH-ordered epitaxial OB1 phase with respect to epitaxial $\mathrm{ZB} \mathrm{SiGe}$ has been discussed by
Martins and Zunger. ${ }^{7}$ Here, the novel result is that the segregation [Fig. 1(c)] into the pseudomorphic $\mathrm{Si}_{4} \mathrm{Ge}_{4}(001)$ superstructure is even more favorable than the $\mathrm{RH}$-ordered epitaxial $\mathrm{OB} 1$ phase with an energy benefit of $4 \mathrm{mRy} / \mathrm{cell}$. The experimental observation ${ }^{2}$ that the ordering is reduced during prolonged annealing may be due to the onset of segregation.

To proceed with the stability analysis of the ordered GaAlAs $_{2}$ phase, we first calculate the energies of separate GaAs and AlAs compounds, but arranged in a tetragonal cell corresponding to that of the (GaAs) - (AlAs) $)_{1}$ superstructure in the [001] orientation. (This is equivalent to the ordered $\mathrm{GaAlAs}_{2}$ compound observed in Ref. 1.) In the next step, we carry out an extensive geometry optimization of the $(\mathrm{GaAs})_{1}-(\mathrm{AlAs})_{1}(001)$ structure by allowing the bonds to relax, and the lattice parameters to vary. The equilibrium total energy is found to be $2.6 \mathrm{mRy} / \mathrm{cell}$ higher than the average of the equilibrium energies of constituent compounds. This implies the metastability of the GaAlAs $s_{2}$ compound. Here, the cause of instability can be sought in the character of the bonds (rather than strain) of the constituent compounds. The GaAs bond is stronger and more ionic ${ }^{8}$ as compared to the AlAs bond, but both have almost equal lengths. In forming the ordered structure, one does not expect much energy benefit by the relaxation of bonds (because they remain essentially unchanged). On the other hand, one loses energy through charge transfer from the more polar bond ( $\mathrm{GaAs}$ ) to the less polar bond (AlAs). In conformity with these arguments, we find the transfer of charge from GaAs to AlAs leading to the loss of energy, but negligibly small gain of energy upon relaxing the bonds.

The metastability of the ordered phase relative to compounds suggests the segregation into GaAs and AlAs in laminar or fine-grained forms. This prediction is supported by the high-resolution cross-sectional bright-field electron micrograph taken from grown films. ' Certainly, the size of the segregation depends on the growth conditions, mainly growth rate, temperature, and composition. Segregated layers do not show any periodic arrangement, and thus may be arbitrarily close to each other. Transition to ordered phase or segregation takes place at about $700^{\circ} \mathrm{C}$ - in spite of the excess entropy of disorder-and ceases at higher temperature. This indicates that either the energy difference leading to instability is sizable (perhaps even larger than calculated values), or the kinetics of the process is controlled by surface effects and diffusion.

Effects of various forms of ordering in the $\mathrm{Si}_{1-x} \mathrm{Ge}_{x}$ strained alloy are shown in Table I. While the higherand lower-lying valence and conduction-band states are not much affected, the relative and absolute positions of the states split off from the band edges undergo significant changes. In earlier papers, 4,9 the effect of strain on the band discontinuity was pointed out. Here, 
TABLE I. Variations of the conduction- and valence-band states at the close proximity of the band edges for various ordered structures of the SiGe system. The disordered $D$ and ordered $\mathrm{OB} 1, \mathrm{OB} 2, \mathrm{Si}_{4} \mathrm{Ge}_{4}$ structures are explained in the text. The lowest conduction-band state $C_{M_{1}}$ at the $M$ point and the highest valence-band state $V_{\Gamma_{1}}$ at the $\Gamma$ point of the $D$ structure are taken as reference levels for conduction and valence-band states, respectively. The energy unit is millielectronvolts.

\begin{tabular}{lcrrr}
\hline \hline & $D$ & $\mathrm{OB} 1$ & $\mathrm{OB} 2$ & $\mathrm{Si}_{4} \mathrm{Ge}_{4}$ \\
\hline$C_{\Gamma_{2}}$ & 288 & 300 & 289 & 201 \\
$C_{\Gamma_{1}}$ & 215 & 144 & 230 & 113 \\
$C_{M_{2}}$ & 91 & 95 & 179 & 79 \\
$C_{M_{1}}$ & 0.0 & -37 & 48 & 57 \\
$V_{\Gamma_{1}}$ & 0.0 & 4 & 26 & 48 \\
$V_{\Gamma_{2}}$ & -4 & -13 & 18 & 7 \\
$V_{\Gamma_{3}}$ & -198 & -192 & -172 & -203 \\
\hline \hline
\end{tabular}

the novel aspect is the change of the band discontinuity as a result of the strain-induced long-range order. The effect of ordering in the $\mathrm{Ga}_{1-x} \mathrm{Al}_{x} \mathrm{As}$ alloy is revealed by study of the electronic structure of $(\mathrm{GaAs})_{1}-(\mathrm{AlAs})_{1}$ (001). As opposed to $(\mathrm{GaAs})_{n}-(\mathrm{AlAs})_{n}$ with $n>2$, we find the $n=1$ structure to be an indirect-band-gap material with a conduction-band minimum at the $R$ point. ${ }^{10}$ It is also expected that the value of the band gap differs from that of the alloy. Remarkable changes occur when the width of segregated layers increases. Such a possibility is demonstrated by calculating the electronic structure of $(\mathrm{GaAs})_{4}-(\mathrm{AlAs})_{4}(001)$ and $\mathrm{Si}_{4} \mathrm{Ge}_{4}(001)$ superlattices.
The distribution of charge for all occupied, as well as for the states split off from the conduction-band minima, is shown in Fig. 2. At the $\Gamma$ point, the lowest conduction-band state is localized in the AlAs region [Fig. 2(c)], whereas the second state $258 \mathrm{meV}$ above is more localized in GaAs and has a resonant character [Fig. 2(d)]. At the $M$ point (which corresponds to the $X$ point of the parent $\mathrm{ZB}$ structure) the first and second band states (which are 72 and $101 \mathrm{meV}$ above the lowest conduction-band state at $\Gamma$ ) are also localized in the AlAs region [Fig. 2(e) and 2(f)]. The highest valenceband state is localized in GaAs. These states can be identified as confined states, and their localization suggests a staggered band lineup shown in Fig. 2(a). The quantum wells produced therefrom occur in AlAs for electrons, and in GaAs for holes.

The consensus ${ }^{11}$ about the order and localization of the conduction-band states at large superlattice periodicity is that the first two states at $\Gamma$ and the lowest state at $M$ are all localized in the GaAs region. The third state at $\Gamma$ has a resonant character with more localization in the AlAs region. This leads to the conventional macroscopic band alignment where the band gap of GaAs is fully accommodated in the AlAs band gap. In agreement with the recent photoluminescence experiments, ${ }^{12}$ our results here point to the fact that the band lineup of GaAs-AlAs is strongly dependent on the superlattice periodicity (quantum size effect in superlattices). Our findings are in agreement with those obtained from a recent empirical pseudopotential calculation. ${ }^{13}$ Apart from being the first SCF charge-density calculations for confined states, our results show clearly that even such a

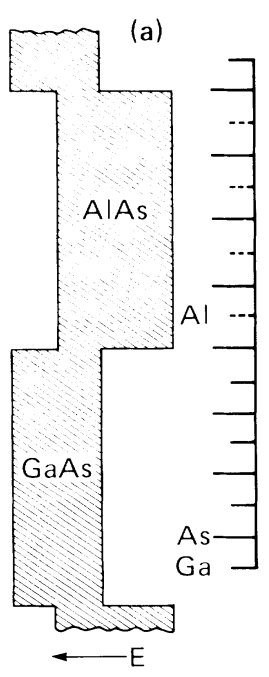

(b)

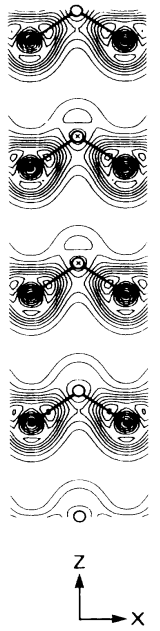

(c)

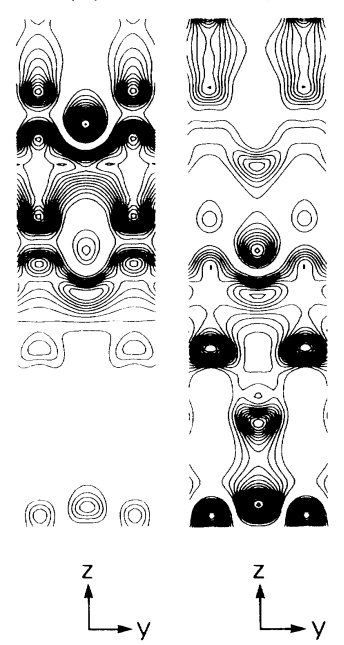

(e)

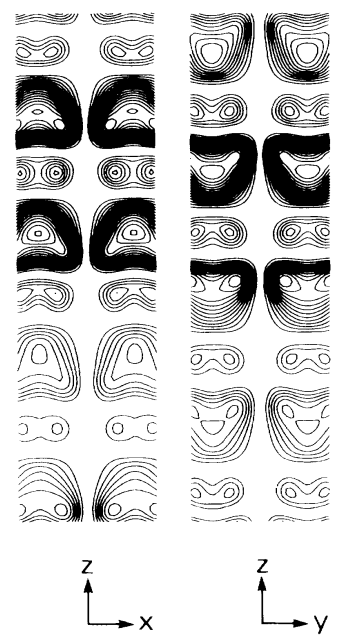

FIG. 2. Charge-density contour plots of the $(\mathrm{GaAs})_{4}-(\mathrm{AlAs})_{4}$ superlattice in the [001] orientation. (a) Energy-gap diagram with bars showing the positions of the $\mathrm{Ga}$, As, and $\mathrm{Al}$ atoms. (b) Total charge density in the (010) plane. (c) Charge distribution of the lowest conduction-band state at the $\Gamma$ point. (d) The second state at the $\Gamma$ point. (e) The first conduction-band state at the $M$ point. (f) The second state at the $M$ point. The contour spacings are 0.01 a.u. for (b), and 0.0002 a.u. for the other panels. 
thin superlattice can support confined states. By examining the planarly averaged SCF potential along the superlattice direction, we also observe that the interface region is rather sharp and only $1-2$ layers thick, leading to 2-3 bilayers of bulk region on both sides. Similar quantumwell formation with confined subbands in minizones is also obtained for the $\mathrm{Si}_{4} \mathrm{Ge}_{4}(001)$ structure which is found to be an indirect-band-gap material. However, because of strain and zone-folding effects the energy separation between direct and indirect band gap is reduced to $\sim 0.1 \mathrm{eV}$.

In conclusion, it can be stated that the laminar segregation with about three layers produces micro-quantum wells in the barrier region causing drastic changes in the optical, electronic, transport, and tunneling properties. If fine grains are separated, they may trap free carriers and have totally confined (quasi-0D) electron or hole states in the potential barrier.

We acknowledge helpful discussions with R. F. C. Farrow, K. Kunc, and G. P. Srivastava. We are particularly thankful to K. A. Müller for his encouragement for pursuing this problem. One of us (I.P.B.) is grateful to T. S. Schneider, K. E. Drangeid, A. Frei, and R. Tomaschett for their constant support while the author was at the Zurich Research Laboratory where this work was completed.

\footnotetext{
(a) Permanent address: Department of Physics, Bilkent University, Anakara, Turkey.

(b) Permanent address: IBM Almaden Research Center, San
}

Jose, CA 95120.

${ }^{1}$ T. S. Kuan, T. F. Kuech, W. I. Wang, and E. L. Wilkie, Phys. Rev. Lett. 54, 201 (1985).

${ }^{2}$ A. Ourmazd and J. C. Bean, Phys. Rev. Lett. 55, 765 (1985).

${ }^{3}$ M. Schlüter, J. R. Chelikowsky, S. G. Louie, and M. L. Cohen, Phys. Rev. B 12, 4200 (1975); references relevant to the method of calculation can be found in I. P. Batra and S. Ciraci, Phys. Rev. B 33, 4312 (1985).

${ }^{4}$ C. G. Van de Walle and R. M. Martin, Phys. Rev. B 34, 5621 (1986).

5I. P. Batra, Phys. Rev. B 29, 7108 (1984); P. N. Keating, Phys. Rev. B 145, 637 (1966).

${ }^{6}$ A. T. Fiory, J. C. Bean, L. C. Feldman, and I. K. Robinson, J. Appl. Phys. 56, 1227 (1984).

7J. L. Martins and A. Zunger, Phys. Rev. Lett. 56, 1400 (1986); see also C. P. Flynn, Phys. Rev. Lett. 57, 599 (1986).

${ }^{8}$ W. A. Harrison and S. Ciraci, Phys. Rev. B 10, 1516 (1974). For an extensive discussion, see G. P. Srivastava, J. L. Martins, and A. Zunger, Phys. Rev. B 31, 2561 (1985).

${ }^{9}$ G. Abstreiter, H. Brugger, T. Wolf, H. Jorke, and H. Herzog, Phys. Rev. Lett. 54, 2441 (1985).

${ }^{10}$ D. M. Bylander and L. Kleinman, Phys. Rev. B 34, 5280 (1986).

${ }^{11}$ D. Ninno, K. B. Wong, M. A. Gell, and M. Jaros, Phys. Rev. B 32, 2700 (1985).

${ }^{12}$ E. Finkman, M. D. Sturge, and M. C. Tamargo, Appl. Phys. Lett. 49, 1299 (1986); P. Dawson, B. A. Wilson, C. W. Tu, and R. C. Miller, Appl. Phys. Lett. 48, 541 (1986); A. Ishibashi, Y. Mori, M. Itabashi, and N. Watanabe, J. Appl. Phys. 58, 2691 (1985).

${ }^{13}$ M. A. Gell, D. Ninno, M. Jaros, and D. C. Herbert, Phys. Rev. B 34, 2416 (1986); also see, J. Ihm, Appl. Phys. Lett. (to be published); I. P. Batra, S. Ciraci, and J. S. Nelson, J. Vac. Sci. Technol. B (to be published). 